\title{
A Repeating Fast Radio Burst
}

\author{
L. G. Spitler ${ }^{1}$, P. Scholz ${ }^{2}$, J. W. T. Hessels ${ }^{3,4}$, S. Bogdanov ${ }^{5}$, A. Brazier ${ }^{6,7}$, F. Camilo ${ }^{5,8}$, S. Chatterjee ${ }^{6}$, \\ J. M. Cordes ${ }^{6}$, F. Crawford ${ }^{9}$, J. Deneva ${ }^{10}$, R. D. Ferdman ${ }^{2}$, P. C. C. Freire ${ }^{1}$, V. M. Kaspi ${ }^{2}$, P. Lazarus ${ }^{1}$, \\ R. Lynch $^{11,12}$, E. C. Madsen ${ }^{2}$, M. A. McLaughlin ${ }^{12}$, C. Patel ${ }^{2}$, S. M. Ransom ${ }^{13}$, A. Seymour ${ }^{14}$, \\ I. H. Stairs ${ }^{15,2}$, B. W. Stappers ${ }^{16}$, J. van Leeuwen ${ }^{3,4} \&$ W. W. Zhu ${ }^{1}$
}

Published online by Nature on 2016 March 2. DOI: 10.1038/nature17168

${ }^{1}$ Max-Planck-Institut für Radioastronomie, Auf dem Hügel 69, B-53121 Bonn, Germany

${ }^{2}$ Department of Physics and McGill Space Institute, McGill University, 3600 University St., Montreal, QC H3A 2T8, Canada

${ }^{3}$ ASTRON, Netherlands Institute for Radio Astronomy, Postbus 2, 7990 AA, Dwingeloo, The Netherlands

${ }^{4}$ Anton Pannekoek Institute for Astronomy, University of Amsterdam, Science Park 904, 1098 XH Amsterdam, The Netherlands

${ }^{5}$ Columbia Astrophysics Laboratory, Columbia University, New York, NY 10027, USA

${ }^{6}$ Department of Astronomy and Space Sciences, Cornell University, Ithaca, NY 14853, USA

${ }^{7}$ Cornell Center for Advanced Computing, Cornell University, Ithaca, NY 14853, USA

${ }^{8}$ Square Kilometre Array South Africa, Pinelands, 7405, South Africa

${ }^{9}$ Department of Physics and Astronomy, Franklin and Marshall College, Lancaster, PA 176043003, USA

${ }^{10}$ Naval Research Laboratory, 4555 Overlook Ave SW, Washington, DC 20375, USA

${ }^{11}$ National Radio Astronomy Observatory, PO Box 2, Green Bank, WV, 24944, USA

${ }^{12}$ Department of Physics and Astronomy, West Virginia University, Morgantown, WV 26506, USA

${ }^{13}$ National Radio Astronomy Observatory, Charlottesville, VA 22903, USA

${ }^{14}$ Arecibo Observatory, HC3 Box 53995, Arecibo, PR 00612, USA

${ }^{15}$ Department of Physics and Astronomy, University of British Columbia, 6224 Agricultural Road Vancouver, BC V6T 1Z1, Canada

${ }^{16}$ Jodrell Bank Centre for Astrophysics, School of Physics and Astronomy, University of Manchester, Manchester, M13 9PL, UK

Fast Radio Bursts are millisecond-duration astronomical radio pulses of unknown physical origin that appear to come from extragalactic distances ${ }^{1+8}$. Previous follow-up observations have failed to find additional bursts at the same dispersion measures (i.e. integrated column density of free electrons between source and telescope) and sky position as the original detections ${ }^{9}$. The apparent non-repeating nature of the fast radio bursts has led several authors to hypothesise that they originate in cataclysmic astrophysical events ${ }^{10}$. Here we report the detection of ten additional bursts from the direction of FRB 121102, using the 305-m Arecibo telescope. These new bursts have dispersion measures and sky positions consistent with the original burst ${ }^{4}$. This unambiguously identifies FRB 121102 as repeating and demonstrates that its source survives the energetic events that cause the bursts. Additionally, the 
bursts from FRB 121102 show a wide range of spectral shapes that appear to be predominantly intrinsic to the source and which vary on timescales of minutes or shorter. While there may be multiple physical origins for the population of fast radio bursts, the repeat bursts with high dispersion measure and variable spectra specifically seen from FRB 121102 support models that propose an origin in a young, highly magnetised, extragalactic neutron $\operatorname{star}^{11,12}$. 
FRB 121102 was discovered ${ }^{4}$ in the PALFA survey, a deep search of the Galactic plane at $1.4 \mathrm{GHz}$ for radio pulsars and fast radio bursts (FRBs) using the 305-m William E. Gordon Telescope at the Arecibo Observatory and the 7-beam Arecibo L-band Feed Array (ALFA) 1314 . The observed dispersion measure (DM) of the burst is roughly three times the maximum value expected along this line of sight in the NE2001 model ${ }^{15}$ of Galactic electron density, i.e. $\beta_{\mathrm{DM}} \equiv$ $\mathrm{DM}_{\mathrm{FRB}} / \mathrm{DM}_{\text {Max }}^{\mathrm{Gal}} \sim 3$, suggesting an extragalactic origin.

Initial Arecibo follow-up observations were limited in both dwell time and sky coverage and resulted in no detection of additional bursts ${ }^{4}$. In 2015 May and June we carried out more extensive follow-up using Arecibo, covering a $\sim 9^{\prime}$ radius with a grid of six ALFA pointings around the then-best sky position of FRB 121102 (Figure 1 and Extended Data Table 1 and 2). As described in the Methods, high-time-resolution, total intensity spectra were recorded, and the data were processed using standard radio-frequency interference (RFI) excision, dispersion removal, and single-pulse-search algorithms implemented in the PRESTO software suite and associated data reduction pipelines ${ }^{14} / 16 / 17$.

We detected 10 additional bursts from FRB 121102 in these observations. The burst properties, and those of the initial FRB 121102 burst, are listed in Table 1. The burst intensities are shown in Figure 2. No other periodic or single-pulse signals of a plausible astrophysical origin were detected at any other DM. Until the source's physical nature is clear, we continue to refer to it as FRB 121102 and label each burst chronologically starting with the original detection.

The ten newly detected bursts were observed exclusively in two adjacent sky positions of the telescope pointing grid located $\sim 1.3^{\prime}$ apart (Figure 1 and Extended Data Table 1 ). The unweighted average $\mathbf{J} 2000$ position from the centres of these two beams is right ascension $\alpha=05^{h} 31^{m} 58^{s}$, declination $\delta=+33^{d} 08^{m} 04^{s}$, with an uncertainty radius of $\sim 3^{\prime}$. The corresponding Galactic longitude and latitude are $l=174.89, b=-0.23$. This more accurate position is $3.7^{\prime}$ from the beam centre of the discovery burst ${ }^{4}$, meaning that FRB 121102 Burst 1 was detected well off axis, as originally concluded.

The measured DMs of all 11 bursts are consistent to within the uncertainties, and the dispersion indices (dispersive delay $\Delta t \propto \nu^{-\xi}$ ) match the $\xi=2.0$ value expected for radio waves traveling through a cold, ionised medium. This is strong evidence that a single astronomical source is responsible for the events. In addition, the $\sim 0.002 \mathrm{DM}$ index uncertainty we calculate for Burst 11 (see Methods) is slightly less than that reported for FRB $110523^{8}$, making this the most precise determination of dispersion index for any FRB thus far. The upper bound on the dispersion index is identical to that of FRB $110523^{8}$, and hence, following the same arguments used there, Burst 11 provides a similar lower limit of $10 \mathrm{AU}$ for the size of the dispersive region.

The 11 bursts have peak flux densities $g S_{1400} \sim 0.02-0.3 \mathrm{Jy}$ at $1.4 \mathrm{GHz}$, where $g$ is the antenna gain at the source's unknown location in the beam normalised to unit amplitude on the beam axis. The other known FRBs typically have order-of-magnitude higher peak flux densities of $g S_{1400} \sim 0.2-2 \mathrm{Jy}$. The wide range of flux densities seen at Arecibo, some near the detection threshold, suggests that weaker bursts are also produced, likely at a higher rate. The rate of burst 
detections is $\sim 3 \mathrm{hr}^{-1}$ for bursts with $g S_{1400} \gtrsim 20 \mathrm{mJy}$ over all observations in which an ALFA beam was within $3.5^{\prime}$ of the improved position. We note, however, that the bursts appear to cluster in time with some observing sessions showing multiple bright bursts and others showing none.

The observed burst full widths at half maximum are $w_{50}=2.8-8.7 \mathrm{~ms}$, which are consistent with the $w_{50}=1.3-23.4 \mathrm{~ms}$ widths seen from other FRBs. No clear evidence for scatter broadening was seen in any of the bursts. Bursts 8 and 10 show double-peaked profiles, which has also been seen in FRB 121002 $2^{7}$. Furthermore, the morphologies of Bursts 8 and 10 evolve smoothly with frequency.

Within our observing band $(1.214-1.537 \mathrm{GHz})$ the burst spectra are remarkably variable. Some are brighter toward higher frequencies, as in the initial discovery, Burst 1, while others are brighter toward lower frequencies. The spectra of Bursts 8 and 10 are not monotonic. The detections of Bursts 6-11 exclusively in Beam 0 of the ALFA receiver (see Extended Data Table 1) means that the bursts must have been detected in the main beam and not in a side-lobe. While the frequency-dependent shape of the main beam attenuates the bursts' intrinsic spectra at higher frequencies if the source is off-axis ${ }^{4}$, this bias is either not large enough or in the wrong direction to cause the observed spectral variability of Bursts 6-11. Given our improved position, Burst 1 is consistent with its detection in a side-lobe, which, unlike in the main beam, could have caused attenuation of the spectrum at lower frequencies. This spectral volatility is reflected by the wide range of spectral indices $\alpha \sim-10$ to +14 obtained from fitting a power-law model $\left(S_{\nu} \propto \nu^{\alpha}\right.$, where $S_{\nu}$ is the flux density at frequency $\nu$ ) to burst spectra (Table 1).

There is no evidence for fine-scale diffractive interstellar scintillation, most likely because it is unresolved by our limited spectral resolution. In principle, the spectra could be strongly modulated if the source is multiply imaged by refraction in the interstellar medium ${ }^{18}$ or by gravitational lensing. However, the splitting angle between sub-images required to produce spectral structure across our band ( $\ll 1$ milli-arcsecond) is much smaller than the expected diffraction angle from interstellar plasma scattering. The fine-scaled diffraction structure in the spectrum will therefore wash out the oscillation. Lastly, positive spectral indices could also be explained by free-free absorption at the source ${ }^{19}$, but this is ruled out by the large spectral differences among bursts. We therefore conclude that the spectral shapes and variations are likely to be predominantly intrinsic to the source.

An analysis of the arrival times of the bursts did not reveal any statistically significant periodicity (see Methods). If the source has a long period ( $\gtrsim 1 \mathrm{~s})$, then it is likely emitting at a

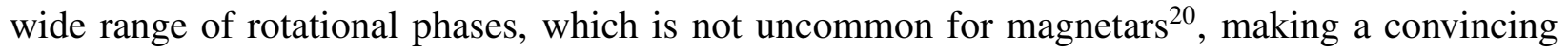
period determination difficult. Due to the small number of detected bursts, we are not sensitive to periodicities much shorter than $\sim 100 \mathrm{~ms}$.

Repeat bursts rule out models involving cataclysmic events - such as merging neutron stars 21 or collapsing supra-massive neutron stars 10 . Bursts from Galactic flare stars have been proposed as a model for FRBs with the DM excess originating in the stellar corona ${ }^{22}$. However, temporal density variations in the corona should produce bursts with varying DMs, which we do not observe. 
Planets orbiting in a magnetised pulsar wind may produce a millisecond-duration burst once per orbital period $^{23}$; however, the observed intra-session separations of our bursts (23 to $572 \mathrm{~s}$ ) are too short to correspond to orbital periods. Repeated powerful radiative bursts are associated with magnetars, and indeed giant flares from the latter have been suggested as a FRB source 12 1924. However, no Galactic magnetar has been seen to emit more than a single giant flare in over four decades of monitoring, arguing against a magnetar giant flare origin for FRB 121102. Magnetars have been observed to exhibit repeating bright radio pulses ${ }^{20}$, however not yet at the energy scale implied if FRB 121102 is more than several hundred kpc away.

Giant pulse emission from an extragalactic pulsar remains a plausible model 11 . The most prominent giant pulses are from the Crab pulsar, which has a large spin-down energy loss rate. Spectral indices calculated from wideband measurements of giant pulses from the Crab pulsar ${ }^{25}$ have a broad distribution ranging from $\alpha \sim-15$ to +10 , as well as frequency "fringes" - i.e. a banded structure to the emission brightness as a function of frequency 26 . These fringes have characteristic widths of a few hundred $\mathrm{MHz}$, and we speculate that - given our 322-MHz observing bandwidth - a similar phenomenon could create the spectral variability we have seen in FRB 121102. The double-peaked nature of some FRB 121102 bursts is also possible in the giant pulse model ${ }^{7}$, and the evolution of these burst morphologies with frequency could imply rapid spectral variation between consecutive (sub-)pulses only milliseconds apart.

The low Galactic latitude and relatively small $\beta_{\mathrm{DM}}$ of FRB 121102 compared with other FRBs raises the question whether it is genuinely extragalactic in origin (see also Methods). However, no $\mathrm{H} \alpha$ or HII regions are seen in archival data along the line of sight to FRB 121102, as might be expected for an intervening ionised nebula ${ }^{4}$ that can give $\beta_{\mathrm{DM}} \gg 1$. Furthermore, a detailed multi-wavelength investigation, which searched for a compact nebula in a sky region that includes the refined position presented here, concluded that FRB 121102's high DM cannot be explained by unmodelled Galactic structure along the line of sight and that FRB 121102 must therefore be extragalactic ${ }^{19}$. Conclusively establishing that FRB 121102 is extragalactic will require arcsecond localisation and association with a host galaxy. The repeating nature of the bursts facilitates such localisation with a radio interferometer.

While the FRB 121102 bursts share many similarities to the FRBs detected using the Parkes $1+3,5-7$ and Green Bank ${ }^{8}$ telescopes, it is unclear whether FRB 121102 is representative of all FRBs. The 10 bursts from FRB 121102 in 2015 were detected near the best-known position in $3 \mathrm{hrs}$ of observations. In contrast, follow-up observations of the Parkes FRBs, again using the Parkes telescope, range in total time per direction from a few hours ${ }^{9}$ to almost 100 hours ${ }^{1}$ and have found no additional bursts. Arecibo's $>10 \times$ higher sensitivity may allow detection of a broader range of the burst-energy distribution of FRBs, thus increasing the chances of detecting repeated bursts; for example, of the 11 bursts from FRB 121102, Parkes may have been capable of detecting only Bursts 8 or 11. More sensitive observations of the Parkes FRBs may therefore show that they also sporadically repeat.

Alternatively, FRB 121102 may be fundamentally different from the FRBs detected at Parkes and Green Bank. As was the case for supernovae and gamma-ray bursts, multiple astrophysical 
processes may be required to explain a diversity of observational properties of FRBs.

1. Lorimer, D. R., Bailes, M., McLaughlin, M. A., Narkevic, D. J. \& Crawford, F. A Bright Millisecond Radio Burst of Extragalactic Origin. Science 318, 777- (2007).

2. Thornton, D. et al. A Population of Fast Radio Bursts at Cosmological Distances. Science 341, 53-56 (2013).

3. Burke-Spolaor, S. \& Bannister, K. W. The Galactic Position Dependence of Fast Radio Bursts and the Discovery of FRB011025. Astrophys. J. 792, 19 (2014).

4. Spitler, L. G. et al. Fast Radio Burst Discovered in the Arecibo Pulsar ALFA Survey. Astrophys. J. 790, 101 (2014).

5. Petroff, E. et al. A real-time fast radio burst: polarization detection and multiwavelength follow-up. Mon. Not. R. Astron. Soc. 447, 246-255 (2015).

6. Ravi, V., Shannon, R. M. \& Jameson, A. A Fast Radio Burst in the Direction of the Carina Dwarf Spheroidal Galaxy. Astrophys. J. 799, L5 (2015).

7. Champion, D. J. et al. Five new Fast Radio Bursts from the HTRU high latitude survey: first evidence for two-component bursts. ArXiv e-prints (2015). 1511.07746.

8. Masui, K. et al. Dense magnetized plasma associated with a fast radio burst. Nature 528, 523-525 (2015).

9. Petroff, E. et al. A survey of FRB fields: limits on repeatability. Mon. Not. R. Astron. Soc. 454, 457-462 (2015).

10. Falcke, H. \& Rezzolla, L. Fast radio bursts: the last sign of supramassive neutron stars. Astron. Astrophys. 562, A137 (2014).

11. Cordes, J. M. \& Wasserman, I. Supergiant Pulses from Extragalactic Neutron Stars. ArXiv:1501:00753 (2015). 1501.00753.

12. Pen, U.-L. \& Connor, L. Local Circumnuclear Magnetar Solution to Extragalactic Fast Radio Bursts. Astrophys. J. 807, 179 (2015).

13. Cordes, J. M. et al. Arecibo Pulsar Survey Using ALFA. I. Survey Strategy and First Discoveries. Astrophys. J. 637, 446-455 (2006).

14. Lazarus, P. et al. Arecibo Pulsar Survey Using ALFA. IV. Mock Spectrometer Data Analysis, Survey Sensitivity, and the Discovery of 40 Pulsars. Astrophys. J. 812, 81 (2015).

15. Cordes, J. M. \& Lazio, T. J. W. NE2001.I. A New Model for the Galactic Distribution of Free Electrons and its Fluctuations. ArXiv Astrophysics e-prints (2002). astro-ph/0207156. 
16. Ransom, S. M. New search techniques for binary pulsars. Ph.D. thesis, Harvard University (2001).

17. Karako-Argaman, C. et al. Discovery and Follow-up of Rotating Radio Transients with the Green Bank and LOFAR Telescopes. Astrophys. J. 809, 67 (2015).

18. Wolszczan, A. \& Cordes, J. M. Interstellar interferometry of the pulsar PSR $1237+25$. Astrophys. J. 320, L35-L39 (1987).

19. Kulkarni, S. R., Ofek, E. O. \& Neill, J. D. The Arecibo Fast Radio Burst: Dense Circum-burst Medium. ArXiv e-prints (2015). 1511.09137.

20. Camilo, F. et al. Transient pulsed radio emission from a magnetar. Nature 442, 892-895 (2006).

21. Hansen, B. M. S. \& Lyutikov, M. Radio and X-ray signatures of merging neutron stars. Mon. Not. R. Astron. Soc. 322, 695-701 (2001).

22. Loeb, A., Shvartzvald, Y. \& Maoz, D. Fast radio bursts may originate from nearby flaring stars. Mon. Not. R. Astron. Soc. 439, L46-L50 (2014).

23. Mottez, F. \& Zarka, P. Radio emissions from pulsar companions: a refutable explanation for galactic transients and fast radio bursts. Astron. Astrophys. 569, A86 (2014).

24. Lyubarsky, Y. A model for fast extragalactic radio bursts. Mon. Not. R. Astron. Soc. 442 , L9-L13 (2014).

25. Karuppusamy, R., Stappers, B. W. \& van Straten, W. Giant pulses from the Crab pulsar. A wide-band study. Astron. Astrophys. 515, A36 (2010).

26. Hankins, T. H. \& Eilek, J. A. Radio Emission Signatures in the Crab Pulsar. Astrophys. J. 670, 693-701 (2007).

Acknowledgements We thank the staff of the Arecibo Observatory, and in particular A. Venkataraman, H. Hernandez, P. Perillat and J. Schmelz, for their continued support and dedication to enabling observations like those presented here. We also thank our commensal observing partners from the Arecibo "Zone of Avoidance" team, in particular T. McIntyre and T. Henning. We also thank M. Kramer for helpful suggestions. The Arecibo Observatory is operated by SRI International under a cooperative agreement with the National Science Foundation (AST-1100968), and in alliance with Ana G. Méndez-Universidad Metropolitana, and the Universities Space Research Association. These data were processed using the McGill University High Performance Computing Centre operated by Compute Canada and Calcul Québec. The National Radio Astronomy Observatory is a facility of the National Science Foundation operated under cooperative agreement by Associated Universities, Inc. The research leading to these results has received funding from the European Research Council (ERC) under the European Union's Seventh Framework Programme (FP7/2007-2013). L.G.S., P.C.C.F. and P.L. gratefully acknowledge financial support from the ERC Starting Grant BEACON under contract no. 279702. J.W.T.H. is an NWO Vidi Fellow and also gratefully acknowledges funding for this work from ERC Starting Grant DRAGNET under contract no. 337062. Work at 
Cornell (J.M.C., S.C., A.B.) was supported by NSF grants AST-1104617 and AST-1008213. V.M.K. holds the Lorne Trottier Chair in Astrophysics and Cosmology and a Canadian Research Chair in Observational Astrophysics and received additional support from NSERC via a Discovery Grant and Accelerator Supplement, by FQRNT via the Centre de Recherche Astrophysique de Québec, and by the Canadian Institute for Advanced Research. J.v.L. acknowledges funding for this research from an ERC Consolidator Grant under contract no. 617199. Pulsar research at UBC is supported by an NSERC Discovery Grant and by the Canadian Institute for Advanced Research.

Author Contributions L.G.S. and J.W.T.H. led the design and execution of the observing campaign described here. P.S. performed the analysis that discovered the radio bursts. More detailed analysis of the signal properties was done by L.G.S., P.S., S.M.R., M.A.M., J.W.T.H., S.C. and J.M.C.. L.G.S., J.W.T.H., P.S. and V.M.K. led the writing of the manuscript. All authors contributed significantly to the interpretation of the analysis results and to the final version of the manuscript.

Competing Interests The authors declare that they have no competing financial interests.

Correspondence Correspondence and requests for materials should be addressed to J.W.T.H. (email: J.W.T.Hessels@uva.nl). 
Table 1: Properties of detected bursts. Uncertainties are the $68 \%$ confidence interval, unless otherwise stated.

\begin{tabular}{lllllll}
\hline \hline No. & $\begin{array}{l}\text { Barycentric } \\
\text { Peak Time (MJD) }\end{array}$ & $\begin{array}{l}\text { Peak Flux } \\
\text { Density }(\mathrm{Jy})^{b}\end{array}$ & $\begin{array}{l}\text { Fluence } \\
(\mathrm{Jy} \mathrm{ms})^{b}\end{array}$ & $\begin{array}{l}\text { Gaussian } \\
\text { Width }^{c}(\mathrm{~ms})\end{array}$ & $\begin{array}{l}\text { Spectral } \\
\text { Index }^{d}\end{array}$ & $\begin{array}{l}\text { DM } \\
\left(\mathrm{pc} \mathrm{cm}^{-3}\right)^{e}\end{array}$ \\
\hline 1 & 56233.282837008 & 0.04 & 0.1 & $3.3 \pm 0.3$ & $8.8 \pm 1.9$ & $553 \pm 5 \pm 2$ \\
2 & 57159.737600835 & 0.03 & 0.1 & $3.8 \pm 0.4$ & $2.5 \pm 1.7$ & $560 \pm 2 \pm 2$ \\
3 & 57159.744223619 & 0.03 & 0.1 & $3.3 \pm 0.4$ & $0.9 \pm 2.0$ & $566 \pm 5 \pm 2$ \\
4 & 57175.693143232 & 0.04 & 0.2 & $4.6 \pm 0.3$ & $5.8 \pm 1.4$ & $555 \pm 1 \pm 2$ \\
5 & 57175.699727826 & 0.02 & 0.09 & $8.7 \pm 1.5$ & $1.6 \pm 2.5$ & $558 \pm 6 \pm 4$ \\
6 & 57175.742576706 & 0.02 & 0.06 & $2.8 \pm 0.4$ & & $559 \pm 9 \pm 1$ \\
7 & 57175.742839344 & 0.02 & 0.06 & $6.1 \pm 1.4$ & $-3.7 \pm 1.8$ & \\
8 & 57175.743510388 & 0.14 & 0.9 & $6.6 \pm 0.1$ & & $556.5 \pm 0.7 \pm 3$ \\
9 & 57175.745665832 & 0.05 & 0.3 & $6.0 \pm 0.3$ & $-10.4 \pm 1.1$ & $557.4 \pm 0.7 \pm 3$ \\
10 & 57175.747624851 & 0.05 & 0.2 & $8.0 \pm 0.5$ & & $558.7 \pm 0.9 \pm 4$ \\
11 & 57175.748287265 & 0.31 & 1.0 & $3.06 \pm 0.04$ & $13.6 \pm 0.4$ & $556.5 \pm 0.1 \pm 1$ \\
\hline \hline
\end{tabular}

${ }^{a}$ Arrival time corrected to the solar-system barycentre and referenced to infinite frequency (i.e the time delay due to dispersion is removed).

${ }^{b}$ Lower limit since it assumes burst is detected at the centre of the beam (i.e. an assumed gain of $10 \mathrm{~K} / \mathrm{Jy}$ yielding a system equivalent flux density of $3 \mathrm{Jy}$ ).

$c$ Widths are the full-width at half maximum.

${ }^{d}$ Bursts 8 and 10 are not well-fit by a power-law model. Burst 6 is too corrupted by RFI to include.

${ }^{e}$ Quoted errors are, in order, statistical and systematic (see Methods). Burst 7 was too weak and corrupted by RFI to include. 


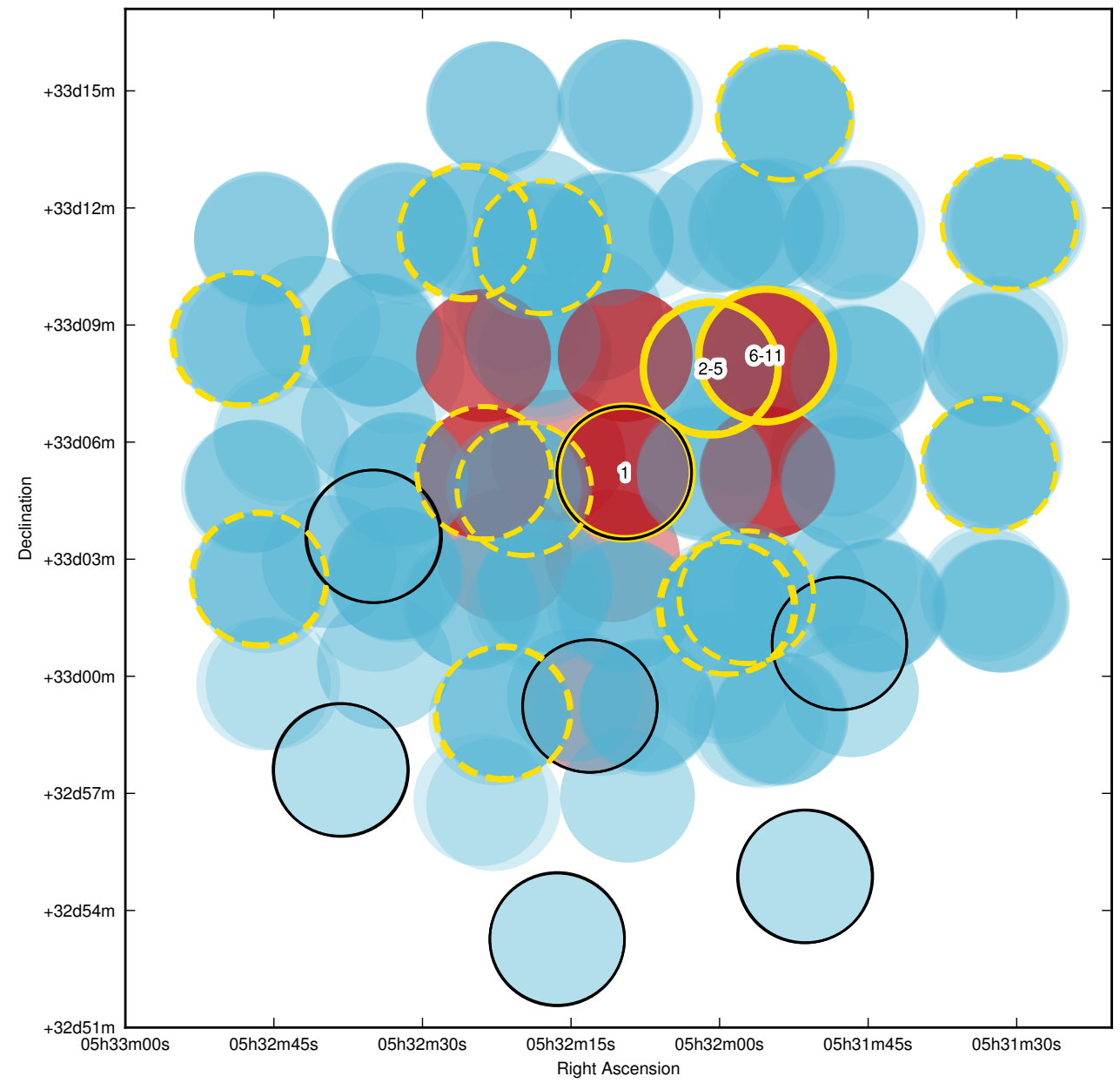


Figure 1 Discovery and follow-up detections of FRB 121102. For each seven-beam ALFA pointing, the central and outer six beams are shown in red and blue, respectively (see Extended Data Table 1 and Table 2). The circles indicate the $\sim 3.5^{\prime}$ half-power widths of the beams at $1.4 \mathrm{GHz}$. Darker shading indicates sky positions with multiple grid observations at roughly the same position. The initial discovery pointing ${ }^{4}$ and second survey observation are outlined in black (these overlap). Beam positions in which bursts were detected are outlined in thick, solid gold (dashed for the other six beams from the same pointing) and the corresponding burst identifier numbers (Table 1) are also given. 


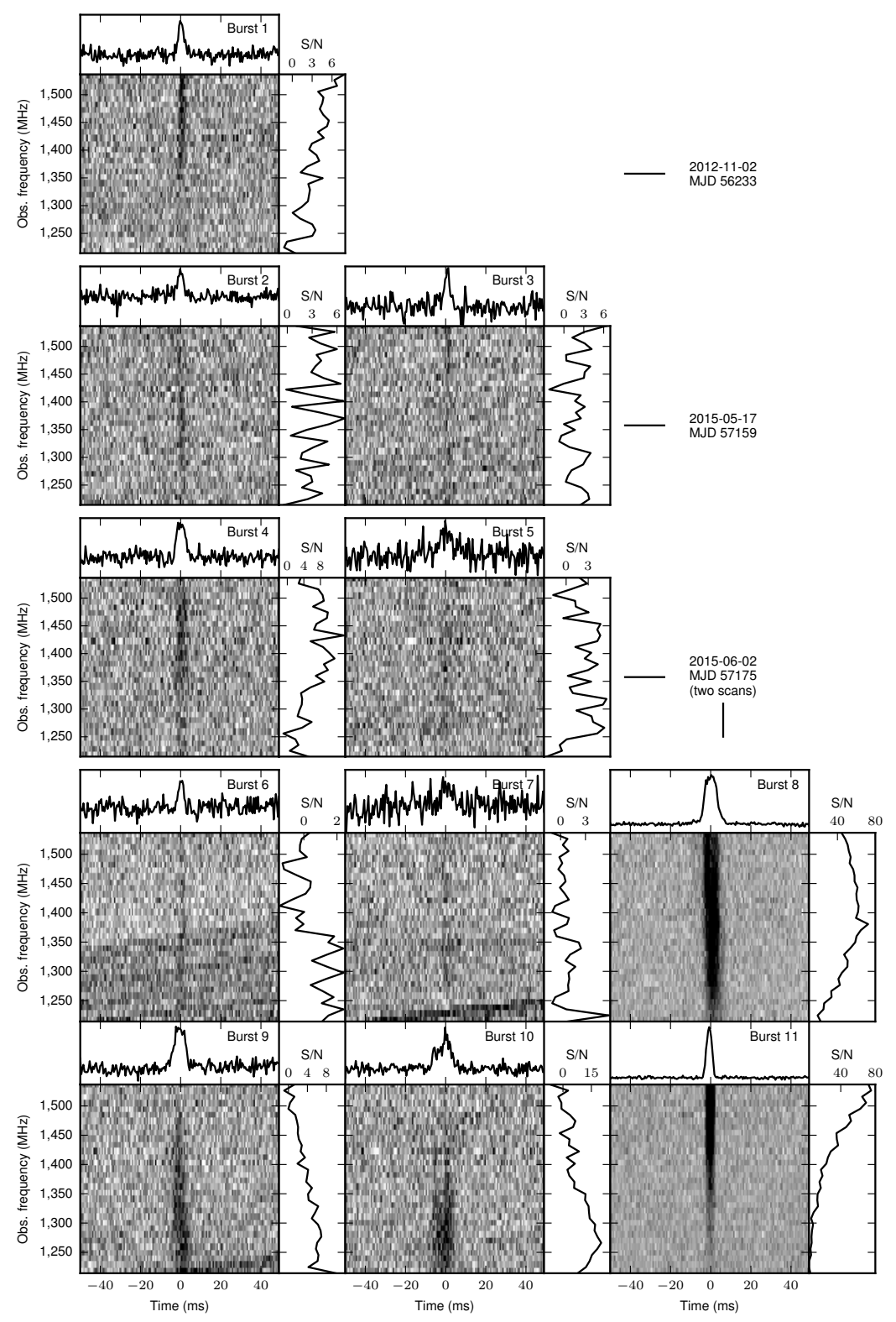

Figure 2 FRB 121102 burst morphologies and spectra. The central greyscale (linearly scaled) panels show the total intensity versus observing frequency and time, after correcting for dispersion to $\mathrm{DM}=559 \mathrm{pc} \mathrm{cm}^{-3}$. The data are shown with $10 \mathrm{MHz}$ and $0.524 \mathrm{~ms}$ frequency and time resolution, respectively. The diagonal striping at low radio frequencies for Bursts 6, 7 and 9 is due to RFI that is unrelated to FRB 121102. The upper sub-panels are burst profiles summed over all frequencies. The band-corrected burst spectra are shown in the right sub-panels. The signal-to-noise scales for the spectra are shown on each sub-panel. All panels are arbitrarily and independently scaled. 


\section{Methods}

\section{Positional gridding strategy and burst localisation}

Extended Data Table 1 summarises all the observations made in both the initial 2013 follow-up and 2015 May/June observations (project code p2886). The ALFA Beam 0 pointing positions in J2000 equatorial coordinates are summarised in Extended Data Table 2. In p2030/p2886 observations, the major axis of the ALFA receiver was rotated $19^{\circ} / 90^{\circ}$ with respect to North ${ }^{4}$.

In 2015 May/June, we searched for additional bursts from FRB 121102 using a grid of six pointings using the seven-beam ALFA receiver to cover a generous $\sim 9^{\prime}$ radius around the discovery beam position and side-lobe. The ALFA receiver was aligned East-West to optimise the sky coverage for this specific purpose. The centre beams of the six grid pointings are shown in red in Figure 1, and the six outer ALFA beams are shown in blue. Each grid pointing position was observed at least four times for $\sim 1000 \mathrm{~s}$. The beam positions of the discovery observation and 2013 follow-up gridding ${ }^{4}$ with ALFA (in that case rotated $19^{\circ}$ with respect to North) are also indicated using the same colour scheme. The outer six ALFA beams in the multiple grid observations are only at roughly the same position because the projection of the ALFA beams on the sky depends on the position of the telescope feed with respect to the primary reflecting dish, and these do not overlap perfectly between independent observations.

Two bursts on May 17 (Bursts 2 and 3) and two on June 2 (Bursts 4 and 5) were detected at a single grid position: FRBGRID2b in ALFA Beam 6, which had positions of $\alpha=05^{h} 32^{m} 01^{s}$, $\delta=+33^{d} 07^{m} 56^{s}$ and $\alpha=05^{h} 32^{m} 01^{s}, \delta=+33^{d} 07^{m} 53^{s}$ (J2000) at the two epochs - i.e. only a few arcseconds apart. Six more bursts (Bursts 6-11) were detected on June 2 at a neighbouring grid position, FRBGRID6b in ALFA Beam $0, \sim 1.3^{\prime}$ away at $\alpha=05^{h} 31^{m} 55^{s}, \delta=+33^{d} 08^{m} 13^{s}$. In all cases bursts were detected in only one beam of the seven-beam ALFA receiver at any given time. This shows that the bursts must originate beyond Arecibo's Fresnel length of $\sim 100 \mathrm{~km}^{19}$.

The intermittency of FRB 121102 makes accurate localisation more challenging. Nonetheless, the detection in adjacent grid positions is informative, and to refine the position of FRB 121102, we simply take the average position between FRBGRID2b ALFA Beam 6 and FRBGRID6b ALFA Beam 0, which gives: $\alpha=05^{h} 31^{m} 58^{s}$, declination $\delta=+33^{d} 08^{m} 04^{s}$ (J2000) and, equivalently, Galactic longitude and latitude $l=174.89, b=-0.23$. The approximate uncertainty radius of $\sim 3^{\prime}$ is based on the amount of overlap between the two detection beam positions and the ALFA beam width at half power, which is $\sim 3.5^{\prime}$. The distance from the initially reported Burst 1 position is $3.7^{\prime}$, consistent with the interpretation that this burst was detected in a side-lobe. Though FRB 121102 bursts have been detected in beams with different central sky positions, all detections are consistent with a well defined sky position when one considers the imprint of the ALFA gain pattern on the sky during each observation ${ }^{4}$. 


\section{Galactic versus extragalactic interpretation}

Noteworthy is the fact that FRB 121102 lies directly in the Galactic plane, whereas the other claimed FRBs lie predominantly at high Galactic latitudes. The PALFA survey is only searching in the Galactic plane, however, and no comparable FRB survey at $1.4 \mathrm{GHz}$ with Arecibo has been done at high Galactic latitudes. Therefore, this difference may simply be a consequence of where Arecibo has most deeply searched for FRBs and does not necessarily suggest that FRB 121102 is of Galactic origin. Furthermore, FRB 121102 was found in the Galactic anti-centre region of the PALFA survey, whereas searches in the inner-Galaxy region have thus far found no FRBs ${ }^{14}$. This may be because the Galactic foregrounds in the anti-centre region are comparatively low, so the deleterious effects of DM smearing and scattering, which may reduce our sensitivity to FRBs, are less important in the outer Galaxy than inner Galaxy.

The low Galactic latitude of FRB 121102 also contributes to its low DM excess factor $\beta_{\mathrm{DM}} \sim 3$ compared to the $\beta_{\mathrm{DM}} \sim 1.2-40$ range seen for the other 15 FRBs in the literature. Only FRB $010621^{27}$, with $\beta_{\mathrm{DM}} \sim 1.2$, has a lower $\beta_{\mathrm{DM}}$ than FRB 121102, and it has been proposed to be Galactic ${ }^{28}$. We note, however, that six of 16 FRBs have DMs comparable to or lower than FRB 121102. Furthermore, its total Galactic DM excess $\mathrm{DM}_{\mathrm{FRB}}-\mathrm{DM}_{\mathrm{Max}}^{\mathrm{Gal}} \sim 370 \mathrm{pc} \mathrm{cm}^{-3}$ is larger than that of the first-discovered $\mathrm{FRB}^{1}$. Lastly, within a generous 20-degree radius of FRB 121102, the highest-DM pulsar known is the millisecond pulsar PSR J0557+1550 29 (also a PALFA discovery), which has $\mathrm{DM}=103 \mathrm{pc} \mathrm{cm}^{-3}$ and $\beta_{\mathrm{DM}}=0.6$, as well as the highest DMinferred distance ${ }^{15}$ of any pulsar in this region, $d=5.7 \mathrm{kpc}$. FRB 121102's DM is clearly anomalous, even when compared to this distant Galactic anti-centre pulsar. At an angular offset of 38 degrees, we note the existence of PSR J0248+6021, with $\mathrm{DM}=370 \mathrm{pc} \mathrm{cm}^{-3}$ and $\beta_{\mathrm{DM}}=1.8$. While the DM of this young, 217-ms pulsar is in excess of the maximum Galactic contribution in the NE2001 model ${ }^{15}$, this can be explained by its location within the dense, giant HII region W5 in the Perseus arm ${ }^{30}$ at a distance of $2 \mathrm{kpc}$. A similar association for FRB 121102 has been sought to explain its $\beta_{\mathrm{DM}} \sim 3$, but multi-wavelength investigations have as yet found no unmodelled Galactic structure ${ }^{419}$. In summary, FRB 121102's comparatively low $\beta_{\mathrm{DM}}$ does not strongly distinguish it from other FRBs, or necessarily suggest it is more likely to be Galactic.

\section{Observations and search processing}

Here we provide a brief description of the Arecibo Mock spectrometer data and search pipeline $e^{14}$ used for our follow-up observations of FRB 121102. The 1.4-GHz data were recorded with the Mock spectrometers, which cover the full ALFA receiver bandwidth in two subbands. Each 172$\mathrm{MHz}$ subband was sampled with 16 bits, a time resolution of $65.5 \mu \mathrm{s}$, and frequency resolution of $0.34 \mathrm{MHz}$ in 512 channels. The data were later converted to 4-bit samples to reduce the data storage requirements. Before processing, the two subbands were combined into a single band of $322 \mathrm{MHz}$ (accounting for frequency overlap between the two subbands), which was centred at $1375 \mathrm{MHz}$ and spans (1214.3 - 1536.7 MHz). 
We used the PALFA PRESTO-based ${ }^{\sqrt{16}}$ search pipeline ${ }^{14}$ to search for astrophysical signals in the frequency and time domains. These data were processed using the McGill University High Performance Computing Centre operated by Compute Canada and Calcul Québec. The presence of radio frequency interference (RFI) can have a detrimental effect on our ability to detect bursts. We therefore applied PRESTO's rfifind to identify contaminated frequency channels and time blocks. Flagged channels and time blocks were masked in subsequent analyses. Time blocks contaminated by RFI are identified using data that are not corrected for dispersive delay (i.e. $\mathrm{DM}=0 \mathrm{pc} \mathrm{cm}^{-3}$ ), in order to avoid removing astrophysical signals. The data were corrected for dispersion using 7292 trial DMs ranging from 0 to $9866.4 \mathrm{pc} \mathrm{cm}^{-3}$, generating a time series at each trial. We performed Fourier analyses of all the time series to look for periodic signals using PRESTO's accelsearch and detected no significant signal of a plausible astrophysical origin.

We searched for single pulses in each dispersion-corrected time series by convolving a template bank of boxcar functions with widths ranging from 0.13 to $100 \mathrm{~ms}$. This optimises the detection of pulses with durations longer than the native sample time of the data. Single-pulse events at each DM were identified by applying a signal-to-noise ratio $(\mathrm{S} / \mathrm{N})$ threshold of 5 .

These single-pulse events were grouped and ranked using the RRATt rap sifting algorithm ${ }^{17}$. An astrophysical pulse is detected with maximum S/N at the signal's true DM and is detected with decreasing S/N at nearby trial DMs. This is not generally the case for RFI, whose S/N does not typically peak at a non-zero trial DM. The RRATtrap algorithm ranks candidates based on this DM behaviour and candidate plots are produced for highly ranked single-pulse groups. These plots display the significance of the pulse as a function of DM and time as well as an image of the signal as a function of time and observing frequency (e.g. Figure 2). The resulting plots were inspected for astrophysical signals, and pulses were found at a DM of $\sim 559 \mathrm{pc} \mathrm{cm}^{-3}$ at a sky position consistent with the discovery position of FRB $121102^{4}$. It is possible that the analysed data contain weaker bursts, which cannot be reliably identified because their $\mathrm{S} / \mathrm{N}$ is too low to distinguish them from RFI or statistical noise. If, in the future, the bursts are shown to have an underlying periodicity, then this would enable a deeper search for weak bursts.

\section{Timing analysis of burst arrival times}

Using several approaches, we searched for an underlying periodicity matching the arrival times of the eight bursts detected in the 2015 June 2 observing session. There are no significant periodicities detected through a standard Fast Fourier Transform of the time series. We then carried out a similar analysis to that routinely used to detect periodicities in sporadically emitting radio pulsars ${ }^{31}$. In this analysis, we calculate differences between all of the burst arrival times and search for the greatest common denominator of these differences. We found several periods, not harmonically related, that fit different subsets of bursts within a tolerance of $1 \%$ of the trial period, but none that fit all of the bursts. We subsequently calculated residuals for the times-of-arrival for the eight bursts detected on 2015 June 2 for a range of trial periods using the pulsar timing packages TEMPO and PINT. We found that some of the periods returned by the differencing algorithm also resulted in 
residuals with root-mean-square (RMS) of less than $1 \%$ of the trial period. However, there were many non-harmonically related candidate periods resulting in residuals of a comparable RMS. Furthermore, given the number of trials necessary for this search, none of these trial periods was statistically significant. In addition, due to the small number of detected bursts, and the widths of the pulses, we were not sensitive to periodicities much shorter than $\sim 100 \mathrm{~ms}$ as our tolerance for a period match (or acceptable RMS) becomes a large fraction of the period and there are many possible fits. The 16-day gap between the 2015 May and June detections precluded us from including the May bursts in any search for periodicity in the single pulses.

\section{Spectral fitting}

In order to produce the spectra shown in the right panels of Figure 2, we corrected each spectrum for the bandpass of the receiver. We estimated the bandpass by taking the average of the raw data samples for each frequency channel. We then median-filtered that average bandpass with a width of 20 channels to remove the effects of narrow-band RFI and divided the observed spectrum of each burst by this median-filtered bandpass. The band-corrected burst spectra shown in the right sub-panels of Figure 2 are still somewhat contaminated by RFI, however. The bottom and top 10 channels $(3.4 \mathrm{MHz})$ of the band were ignored due to roll-off in the receiver response.

To characterise the bandpass-corrected spectrum of each burst, we applied a power-law model using least-squares fitting. The power-law model is described by $S_{\nu} \propto \nu^{\alpha}$, where $S_{\nu}$ is the flux density in a frequency channel, $\nu$ is the observing frequency, and $\alpha$ is the spectral index. These measured spectral indices and their uncertainties are shown in Table 1 . We do not include a spectral index value for Burst 6 because of the RFI in the lower half of the band. For Bursts 7 and 9, we exclude data below $1250 \mathrm{MHz}$, because of RFI contamination. For Bursts 8 and 10, the power-law model was not a good descriptor, and therefore no value is reported in Table 1 .

Note, we verified this technique by applying the bandpass correction to PALFA data of pulsar B1900+01. The measured spectral index was calculated for ten, bright single pulses, and the values are consistent with the published value.

\section{Measurement of dispersion measure and index}

We measured the DM for 10 of the 11 bursts and additionally the dispersion index (dispersive delay $\Delta t \propto \nu^{-\xi}$ ) for the brightest two. The DM and DM index, $\xi$, were calculated with a leastsquares routine using the SIMPLEX and MIGRAD functions from the CERN MINUIT package (http://www.cern.ch/minuit). The user specifies the assumed form of the intrinsic pulse shape, which is then convolved with the appropriate DM smearing factor. For these fits a boxcar pulse template was used.

Subbanded pulse profiles for each burst were generated by averaging blocks of frequency 
channels (i.e. frequency scrunching). The number of subbands generated depended on the $\mathrm{S} / \mathrm{N}$ of the burst to ensure that there was sufficient $\mathrm{S} / \mathrm{N}$ in each subband for the fit to converge. Subbands with no signal were excluded from the fit. Furthermore, the data were binned in time to further increase the $\mathrm{S} / \mathrm{N}$ and reduce the effects of frequency-dependent flux evolution. As the true intrinsic pulse width is not known, each burst was fit with a range of boxcar widths. The parameters corresponding to the input template yielding the cleanest residuals are reported.

The DM value was fit keeping the DM index fixed at 2.0. Note, Burst 7 was too weak and corrupted by RFI to obtain reasonable fits. Additionally, for the brightest two bursts (8 and 11), we also did a joint fit of DM and DM index. The resulting DM index fits were $2.00 \pm 0.02$ and $1.999 \pm 0.002$ for Burst 8 and 11, respectively. These values are as expected for radio waves traveling through a cold, ionised medium

Frequency-dependent pulse profile evolution introduces systematic biases into the times of arrival in each subband. These biases in turn bias the DM determination. These systematics cannot be mitigated without an accurate model for the underlying burst shape versus frequency, which is not available in this case, and is further complicated by the fact that the burst morphology also changes randomly from burst to burst. We estimated the systematic uncertainty by considering what DM value would produce a delay across our observing band that is comparable to half the burst width in each case.

Table 1 presents the results of the fits with the statistical and systematic uncertainties both quoted. The DM estimates do not include barycentric corrections (of order 0.01 to $0.1 \mathrm{pc} \mathrm{cm}^{-3}$ ). Although FRB 121102 is close to the ecliptic, the angular separation from the Sun was always much larger than 10 degrees, and any annual contribution to the DM from the solar wind was small $\left(<10^{-3} \mathrm{pc} \mathrm{cm}^{-3}\right) 3233$. These effects are, therefore, much smaller than the aforementioned systematics in modelling the DMs of the bursts.

The $\pm 1-\sigma$ range of DMs for the 10 new bursts is $558.1 \pm 3.3 \mathrm{pc} \mathrm{cm}^{-3}$, consistent with the discovery value ${ }^{4}, 557.4 \pm 2.0 \mathrm{pc} \mathrm{cm}^{-3}$. The DMs and DM index reported here and previously ${ }^{4}$ were calculated using different methods. These two approaches fit for different free parameters, so different co-variances between parameters may result in slightly different values. Also, different time and frequency resolutions were used. Nonetheless, the Burst 1 parameters quoted here and previously 4 are consistent within the uncertainties. The consistency of the DMs is conclusive evidence that a single source is responsible for the events.

\section{The role of interstellar scattering}

Some FRBs have shown clear evidence for multi-path propagation from scattering by the intervening interstellar or extragalactic material along the line of sight ${ }^{26-8}$. However, the burst profiles from FRB 121102 show no obvious evidence for asymmetry from multi-path propagation. An upper bound on the pulse broadening time from Burst 1 is $1.5 \mathrm{~ms}$ at $1.5 \mathrm{GHz}^{4}$. Using the NE2001 model for a source far outside the Galaxy, the expected pulse broadening is $\sim 20 \mu \mathrm{s} \nu^{-4.4}$ with $\nu$ 
in $\mathrm{GHz}$, an order of magnitude smaller than the $\sim 2 \mathrm{~ms}$ pulse widths and $\sim 0.7 \mathrm{~ms}$ intra-channel dispersion smearing. The features of the spectra can not be explained by diffractive interstellar scintillations; the predicted scintillation bandwidth for FRB 121102 is $\sim 50 \mathrm{kHz}$ at $1.5 \mathrm{GHz}$, which is unresolved by the $0.34 \mathrm{MHz}$ frequency channels of our data. We would, therefore, also not expect to observe diffractive interstellar scintillation in our bursts. Additional scattering occurring in a host galaxy and the intergalactic medium is at a level below our ability to detect. However, observations at frequencies below $1.5 \mathrm{GHz}$ may reveal pulse broadening that is not substantially smaller than the upper bound if we use as a guide the observed pulse broadening from other $\mathrm{FRBs}^{2} 6-8.6 \mathrm{Fu}-$ ture observations that quantify diffractive interstellar scintillations can provide constraints on the location of extragalactic scattering plasma relative to the source, as demonstrated for FRB $110523^{8}$.

The upper bound on pulse broadening for FRB 121102 implies that the apparent, scattered source size for radio waves incident on the Milky Way's ISM is small enough so that refractive interstellar scintillation (RISS) from the ISM is expected. For the line of sight to FRB 121102 we use the NE2001 model to estimate an effective scattering-screen distance of $\sim 2 \mathrm{kpc}$ from Earth and a scattering diameter of 6 mas. The implied length scale for phase-front curvature is then $l_{\text {RISS }} \sim$ $2 \mathrm{kpc} \times 6 \mathrm{mas}=12 \mathrm{AU}$. For an effective, nominal velocity, $V_{\text {eff }}=100 V_{100} \mathrm{~km} \mathrm{~s}^{-1}$, the expected RISS time scale is $\Delta t_{\mathrm{RISS}}=l_{\mathrm{ISS}} / V_{\text {eff }}=215 V_{100}^{-1} \nu_{1 \mathrm{GHz}}^{-2.2}$ days. At $1.5 \mathrm{GHz}$ and an effective velocity due to Galactic rotation, which is about $200 \mathrm{~km} \mathrm{~s}^{-1}$ in the direction of FRB 121102, RISS time scales of 20-40 days are expected. Modulation from RISS can be several tens of percent ${ }^{34}$. This could play a role in the detections of bursts in 2015 mid-May and 2015 June and their absence in 2015 early-May and at other epochs. However, the solar system and the ionised medium have the same Galactic rotation, so the effective velocity could be smaller than $100 \mathrm{~km} \mathrm{~s}^{-1}$, leading to longer RISS time scales.

\section{Data availability}

The beam positions used in Figure 1 are available as a text file. The data of the bursts used to generate Figure 2 are provided as a text file.

\section{Code availability}

The code used to analyse the data is available at the following sites:

PRESTO (https://github.com/scottransom/presto), RRATtrap (https://github.com/ckarako/RRATtrap), TEMPO (http://tempo.sourceforge.net/), and PINT (http://github.com/nanograv/PINT).

27. Keane, E. F., Stappers, B. W., Kramer, M. \& Lyne, A. G. On the origin of a highly dispersed coherent radio burst. Mon. Not. R. Astron. Soc. 425, L71-L75 (2012).

28. Bannister, K. W. \& Madsen, G. J. A Galactic origin for the fast radio burst FRB010621. Mon. Not. R. Astron. Soc. 440, 353-358 (2014). 
29. Scholz, P. et al. Timing of Five Millisecond Pulsars Discovered in the PALFA Survey. Astrophys. J. 805, 85 (2015).

30. Theureau, G. et al. PSRs J0248+6021 and J2240+5832: young pulsars in the northern Galactic plane. Discovery, timing, and gamma-ray observations. Astron. Astrophys. 525, A94 (2011).

31. McLaughlin, M. A. et al. Transient radio bursts from rotating neutron stars. Nature 439, 817-820 (2006).

32. You, X. P., Coles, W. A., Hobbs, G. B. \& Manchester, R. N. Measurement of the electron density and magnetic field of the solar wind using millisecond pulsars. Mon. Not. R. Astron. Soc. 422, 1160-1165 (2012).

33. The NANOGrav Collaboration, The NANOGrav Nine-year Data Set: Observations, Arrival Time Measurements, and Analysis of 37 Millisecond Pulsars. Astrophys. J. 813, 65 (2015).

34. Rickett, B. J. Radio propagation through the turbulent interstellar plasma. Ann. Rev. Astron. Astrophys. 28, 561-605 (1990). 
Extended Data 
Extended Data Table 1: Arecibo FRB 121102 discovery and follow-up observations. The observing setup of these observations is described in the Methods.

\begin{tabular}{|c|c|c|c|c|c|c|c|}
\hline UTC Date & Project & Position & Receiver & $\begin{array}{l}\text { Frequency } \\
(\mathrm{GHz})\end{array}$ & Backend & $\begin{array}{l}\text { Dwell time } \\
\text { (s) }\end{array}$ & \# Bursts \\
\hline \multicolumn{8}{|c|}{ Survey discovery observations presented in Spitler et al. (2014) } \\
\hline 2012-11-02 & p2030 & FRBDISC & ALFA & 1.4 & Mocks & 200 & 1 (Beam4) \\
\hline 2012-11-04 & p2030 & FRBDISC & ALFA & 1.4 & Mocks & 200 & 0 \\
\hline \multicolumn{8}{|c|}{ Follow-up observations presented in Spitler et al. (2014) } \\
\hline 2013-12-09 & p2886 & FRBGRID1a & ALFA & 1.4 & Mocks & 2700 & 0 \\
\hline 2013-12-09 & p2886 & FRBGRID2a & ALFA & 1.4 & Mocks & 970 & 0 \\
\hline 2013-12-09 & p2886 & FRBGRID2a & ALFA & 1.4 & Mocks & 1830 & 0 \\
\hline 2013-12-10 & p2886 & FRBGRID3a & ALFA & 1.4 & Mocks & 2700 & 0 \\
\hline 2013-12-10 & p2886 & FRBDISC & $327-\mathrm{MHz}$ & 0.327 & PUPPI & 2385 & 0 \\
\hline \multicolumn{8}{|c|}{ Follow-up observations presented here for the first time } \\
\hline 2015-05-02 & p2886 & FRBDISC & L-wide & 1.4 & PUPPI & 7200 & 0 \\
\hline 2015-05-03 & p2886 & FRBGRID1b & ALFA & 1.4 & Mocks & 1502 & 0 \\
\hline 2015-05-03 & p2886 & FRBGRID2b & ALFA & 1.4 & Mocks & 1502 & 0 \\
\hline 2015-05-03 & p2886 & FRBGRID3b & ALFA & 1.4 & Mocks & 343 & 0 \\
\hline 2015-05-03 & p2886 & FRBGRID3b & ALFA & 1.4 & Mocks & 1502 & 0 \\
\hline 2015-05-03 & p2886 & FRBGRID1b & ALFA & 1.4 & Mocks & 921 & 0 \\
\hline 2015-05-05 & p2886 & FRBGRID1b & ALFA & 1.4 & Mocks & 1002 & 0 \\
\hline 2015-05-05 & p2886 & FRBGRID2b & ALFA & 1.4 & Mocks & 1002 & 0 \\
\hline $2015-05-05$ & p2886 & FRBGRID3b & ALFA & 1.4 & Mocks & 1002 & 0 \\
\hline 2015-05-05 & p2886 & FRBGRID4b & ALFA & 1.4 & Mocks & 1002 & 0 \\
\hline $2015-05-05$ & p2886 & FRBGRID5b & ALFA & 1.4 & Mocks & 1002 & 0 \\
\hline 2015-05-05 & p2886 & FRBGRID6b & ALFA & 1.4 & Mocks & 1002 & 0 \\
\hline 2015-05-09 & p2886 & FRBGRID1b & ALFA & 1.4 & Mocks & 1002 & 0 \\
\hline 2015-05-09 & p2886 & FRBGRID2b & ALFA & 1.4 & Mocks & 1002 & 0 \\
\hline 2015-05-09 & p2886 & FRBGRID3b & ALFA & 1.4 & Mocks & 1002 & 0 \\
\hline 2015-05-09 & p2886 & FRBGRID4b & ALFA & 1.4 & Mocks & 1002 & 0 \\
\hline 2015-05-09 & p2886 & FRBGRID5b & ALFA & 1.4 & Mocks & 1002 & 0 \\
\hline 2015-05-09 & p2886 & FRBGRID6b & ALFA & 1.4 & Mocks & 1002 & 0 \\
\hline 2015-05-09 & p2886 & FRBGRID6b & ALFA & 1.4 & Mocks & 425 & 0 \\
\hline 2015-05-17 & p2886 & FRBGRID1b & ALFA & 1.4 & Mocks & 1002 & 0 \\
\hline 2015-05-17 & p2886 & FRBGRID2b & ALFA & 1.4 & Mocks & 1002 & 2 (Beam6) \\
\hline 2015-05-17 & p2886 & FRBGRID3b & ALFA & 1.4 & Mocks & 1002 & 0 \\
\hline 2015-05-17 & p2886 & FRBGRID4b & ALFA & 1.4 & Mocks & 707 & 0 \\
\hline 2015-05-17 & p2886 & FRBGRID4b & ALFA & 1.4 & Mocks & 391 & 0 \\
\hline $2015-05-17$ & p2886 & FRBGRID5b & ALFA & 1.4 & Mocks & 1002 & 0 \\
\hline $2015-05-17$ & p2886 & FRBGRID6b & ALFA & 1.4 & Mocks & 1002 & 0 \\
\hline 2015-06-02 & p2886 & FRBGRID1b & ALFA & 1.4 & Mocks & 1002 & 0 \\
\hline 2015-06-02 & p2886 & FRBGRID2b & ALFA & 1.4 & Mocks & 1002 & 2 (Beam6) \\
\hline 2015-06-02 & p2886 & FRBGRID3b & ALFA & 1.4 & Mocks & 1002 & 0 \\
\hline 2015-06-02 & p2886 & FRBGRID4b & ALFA & 1.4 & Mocks & 1002 & 0 \\
\hline 2015-06-02 & p2886 & FRBGRID5b & ALFA & 1.4 & Mocks & 1002 & 0 \\
\hline 2015-06-02 & p2886 & FRBGRID6b & ALFA & 1.4 & Mocks & 1002 & 6 (Beam0) \\
\hline 2015-06-02 & p2886 & FRBGRID6b & ALFA & 1.4 & Mocks & 300 & 0 \\
\hline
\end{tabular}


Extended Data Table 2: FRB 121102 gridding positions. The J2000 ALFA Beam 0 positions are listed.

\begin{tabular}{lll}
\hline \hline Grid ID & Right Ascension & Declination \\
\hline FRBDISC & $05^{h} 32^{m} 09^{s}$ & $+33^{d} 05^{m} 13^{s}$ \\
FRBGRID1a & $05^{h} 32^{m} 16^{s}$ & $+33^{d} 05^{m} 39^{s}$ \\
FRBGRID2a & $05^{h} 32^{m} 22^{s}$ & $+33^{d} 03^{m} 06^{s}$ \\
FRBGRID3a & $05^{h} 32^{m} 11^{s}$ & $+33^{d} 03^{m} 06^{s}$ \\
FRBGRID1b & $05^{h} 32^{m} 10^{s}$ & $+33^{d} 05^{m} 13^{s}$ \\
FRBGRID2b & $05^{h} 32^{m} 24^{s}$ & $+33^{d} 05^{m} 13^{s}$ \\
FRBGRID3b & $05^{h} 31^{m} 55^{s}$ & $+33^{d} 05^{m} 13^{s}$ \\
FRBGRID4b & $05^{h} 32^{m} 10^{s}$ & $+33^{d} 08^{m} 13^{s}$ \\
FRBGRID5b & $05^{h} 32^{m} 24^{s}$ & $+33^{d} 08^{m} 13^{s}$ \\
FRBGRID6b & $05^{h} 31^{m} 55^{s}$ & $+33^{d} 08^{m} 13^{s}$ \\
\hline \hline
\end{tabular}

